\title{
Cambios cefalométricos obtenidos con activador abierto elástico de Klammt como tratamiento temprano de maloclusión clase II
}

\author{
Jesús Alberto Hernández-Silva*, Esp. ${ }_{1}$, Carolina Rodríguez-Manjarrés, Esp. ${ }_{1}$, \\ Natalia Molina-Betancourt, Od.
}

${ }_{1}$ Universidad del Valle, Cali, Colombia

Recibido: 11 de febrero del 2016 Aprobado: 4 de octubre del 2016

*Autor de correspondencia: Jesús Alberto Hernández-Silva. Calle 3 n. ${ }^{\circ}$ 36B-00, Edificio 132, Escuela de Odontología Facultad de Salud, Universidad del Valle, San Fernando, Cali, Valle del Cauca, Colombia.

Correo electrónico: sualberto@msn.com

Cómo citar este artículo: Hernández-Silva JA, Rodríguez-Manjarrés C, Molina-Betancourt N. Cambios cefalométricos obtenidos con activador abierto elástico de Klammt como tratamiento temprano de maloclusión clase II. Rev Nac Odontol. 2017;13(25):1-9. doi: http://dx.doi.org/10.16925/od.v13i25.1704

Resumen. Introducción: la maloclusión clase II presenta la mayor prevalencia entre las alteraciones dentoesqueléticas a nivel mundial. En Colombia tiene una prevalencia del 74\%. Actualmente, uno de los fundamentos para la realización de tratamientos oportunos es la respuesta rápida de las estructuras óseas y neuromusculares cuanto más joven sea el organismo. Objetivo: identificar los cambios en el complejo craneofacial por medio de radiografías laterales de once pacientes con maloclusión clase II división 1, quienes fueron sometidos a tratamiento de ortopedia funcional con Activador Abierto Elástico de Klammt (AAEK). Metodología: se seleccionó una muestra de once pacientes con maloclusión clase II división 1 entre 8 y 12 años de edad, a los cuales se les realizó tratamiento ortopédico funcional. Los pacientes seleccionados requerían de una radiografía lateral antes de iniciar el tratamiento y una al año; se analizaron los cambios del complejo craneofacial de los pacientes sometidos a tratamiento. Resultados: en los once pacientes se logró interceptar de manera oportuna la clase II división 1 . Se percibe una disminución estadísticamente significativa del ANB, 1/Bap, 1 sup/1 inf y overjet. Conclusión: en este estudio se observó que el AAEK en el tratamiento de la maloclusión clase II división 1 produjo inhibición del crecimiento anterior del área dentoalveolar superior, verticalización de incisivos superiores, restricción de la migración anterior de los molares superiores y posicionamiento anterior del punto B.

Palabras clave: activador abierto elástico de Klammt, cefalometría, maloclusión clase II, tratamiento oportuno. 


\title{
Cephalometric changes induced by Klammt's Elastic Open Activator as early treatment for Class II malocclusion
}

\begin{abstract}
Introduction: Class II malocclusion has the highest prevalence among dentoskeletal alterations worldwide. In Colombia its prevalence is $74 \%$. Currently, one of the reason for administering timely treatments is the rapid response of bone and neuromuscular structures the younger the organism. Aim: To identify changes in the craniofacial complex by means of lateral X-rays of 11 patients with Class II Division 1 malocclusion, who underwent functional orthopedic treatment with Klammt's Elastic Open Activator (KEOA). Method: We selected a sample of 11 patients with Class II Division 1 malocclusion between 8 and 12 years old, who underwent functional orthopedic treatment. The selected patients required a lateral $\mathrm{x}$-ray before starting the treatment and once a year thereafter; the craniofacial complex changes of the patients undergoing treatment were analyzed. Results: In all 11 patients, Class II Division 1 malocclusion was intercepted in a timely manner. A statistically significant decrease in ANB, 1/Bap, $1 \mathrm{sup} / 1 \mathrm{inf}$, and overjet was perceived. Conclusion: In this study it was observed that KEOA in the treatment of Class II Division 1 malocclusion produced inhibited anterior growth of the upper dentoalveolar area, verticalization of upper incisors, restricted anterior migration of upper molars, and anterior positioning of point $B$.
\end{abstract}

Keywords: Klammt's Elastic Open Activator, cephalometry, Class II malocclusion, timely treatment.

\section{Mudanças cefalométricas obtidas com ativador aberto elástico de Klammt como tratamento precoce de má oclusão classe II}

Resumo. Introdução: a má oclusão classe II apresenta a maior prevalência entre as alterações dentoesqueléticas no mundo. Na Colômbia, tem uma prevalência de $74 \%$. Atualmente, um dos fundamentos para a realização de tratamentos precoces é a resposta rápida das estruturas ósseas e neuromusculares quanto mais jovem for o organismo. Objetivo: identificar as mudanças no complexo craniofacial por meio de radiografias laterais de 11 pacientes com má oclusão classe II divisão 1, que foram submetidos a tratamento de ortopedia funcional com Ativador Aberto Elástico de Klammt (AAEK). Metodologia: selecionou-se uma amostra de 11 pacientes com má oclusão classe II divisão 1 entre 8 e 12 anos de idade, nos quais foi realizado tratamento ortopédico funcional. Os pacientes selecionados necessitavam de uma radiografia lateral antes de iniciar o tratamento e uma por ano; foram analisadas as mudanças do complexo craniofacial dos pacientes submetidos ao tratamento. Resultados: nos 11 pacientes, foi possível interceptar de maneira oportuna a má oclusão classe II divisão 1. Percebe-se uma diminuição estatisticamente significativa do ANB, 1/Bap, $1 \mathrm{sup} / 1 \mathrm{inf}$ e overjet. Conclusão: neste estudo, observou-se que o AAEK no tratamento de má oclusão classe II divisão 1 produziu inibição do crescimento anterior da área dentoalveolar superior, verticalização de incisivos superiores, restrição da migração anterior dos molares superiores e posicionamento anterior do ponto B.

Palavras-chave: ativador aberto elástico de Klammt, cefalometria, má oclusão tipo classe II, tratamento precoce. 


\section{Introducción}

La maloclusión clase II se caracteriza por presentar un retrognatismo mandibular y/o prognatismo maxilar que puede afectar la relación molar [1]; dentalmente se encuentra que el surco mesial del primer molar inferior permanente articula por detrás de la cúspide mesovestibular del primer molar superior permanente [2]. Dentro de esta maloclusión, según su componente dental, existe la clase II división 1 (II-1), caracterizada por la vestibularización de todos los incisivos superiores, y la clase II división 2 (II-2), en la que los incisivos centrales superiores están retroinclinados y los incisivos laterales superiores están proinclinados [3-5].

La herencia es probablemente el primer factor etiológico de esta maloclusión; factores adicionales locales como respiración oral, succión digital y deglución disfuncional, entre otros hábitos, son elementos contribuyentes más que desencadenantes en el desarrollo de la maloclusión [6]. Por lo tanto, el control de dichas disfunciones es parte fundamental en el resultado y estabilidad de las diferentes opciones terapéuticas empleadas para su corrección $[7,8]$.

La maloclusión clase is presenta la mayor prevalencia entre las alteraciones dentoesqueléticas a nivel mundial. En Norteamérica, un tercio de la población presenta esta maloclusión [9]; en Colombia, se encuentra una prevalencia del 74\% [10].

Esta maloclusión se manifiesta con diversas alteraciones esqueléticas y/o dentales de acuerdo con su división. La clase II-1 presenta un biotipo dólicofacial o mesofacial, convexidad facial aumentada, altura de la rama corta, dimensión vertical aumentada, incisivos superiores vestibularizados, labios protruidos, labio superior hipotónico e inferior hipertónico e invertido, plano oclusal funcional inclinado/elevado, ángulo de la profundidad facial disminuido y plano mandibular aumentado [11].

Como se mencionó antes, puede comprometer tanto el maxilar como la mandíbula alterando las relaciones esqueléticas en sentido anteroposterior, ya sea por un crecimiento horizontal excesivo del maxilar y deficiente de la mandíbula y/o por una combinación de ambos. Estas maloclusiones presentan asimismo afectación del plano vertical, lo que resulta en una rotación anterior o posterior mandibular [12].
El tratamiento oportuno de las maloclusiones es cada día más frecuente. Uno de los fundamentos para hacerlo es la respuesta rápida de las estructuras óseas y neuromusculares, ya que la mejor respuesta biológica al tratamiento se logra durante los primeros años de vida [13]. La importancia del tratamiento oportuno es que permite recuperar un desarrollo armónico del complejo craneofacial y dental, simplifica los procedimientos correctivos y trae grandes beneficios que conducen al paciente hacia un desarrollo dentofacial favorable $[13,14]$.

Dicho tratamiento se puede llevar a cabo a través de una amplia variedad de aparatología ortopédica funcional que permite, de una manera rápida y eficiente, solucionar las diferentes alteraciones maxilares que acompañan esta maloclusión y corregir la relación intermaxilar $[1,15]$.

Estudios experimentales en ratas y empleando un aparato funcional, realizados por Petrovic [16], demostraron la activación de una serie de fenómenos histológicos y bioquímicos que promueven el crecimiento condilar y la readaptación anteroinferior de la fosa glenoidea, con lo que facilitan una posición más adelantada de la mandíbula (o también llamado avance mandibular) [17, 18].

La aparatología empleada en la corrección de la maloclusión clase in puede ser fija o removible y trabaja mediante la estimulación de los músculos de la masticación y tejidos blandos. Entre los más reconocidos están: Activador, Bionator, Twin Block, Herbst, Headgear, aparatología Frankel y Simoes Network, entre otros $[1,19]$.

El Activador Abierto Elástico de Klammt (AAEK), desarrollado por Georg Klammt, es un aparato ortopédico funcional bimaxilar que induce el posicionamiento anterior de la mandíbula, estimula la actividad de los músculos faciales y genera el engranaje molar deseado en clase I, promueve la expansión de los arcos dentales, mejora la forma del arco y la alineación de los dientes anteriores a través de los arcos vestibulares y los resortes palatinos y linguales, lo cual crea estabilidad favorable para el cambio de postura terapéutico [1, 20-22] (Figura 1).

El AAEK se diferencia de los otros activadores porque elimina la parte acrílica que cubre el paladar y la pared anterior uniendo los dos componentes laterales con un resorte tipo coffin, con lo que brinda un espacio adecuado para la lengua que permite el contacto de esta con el paladar. Así, el volumen del aparato se reduce a elementos funcionales básicos y gracias a esta pérdida de volumen, 


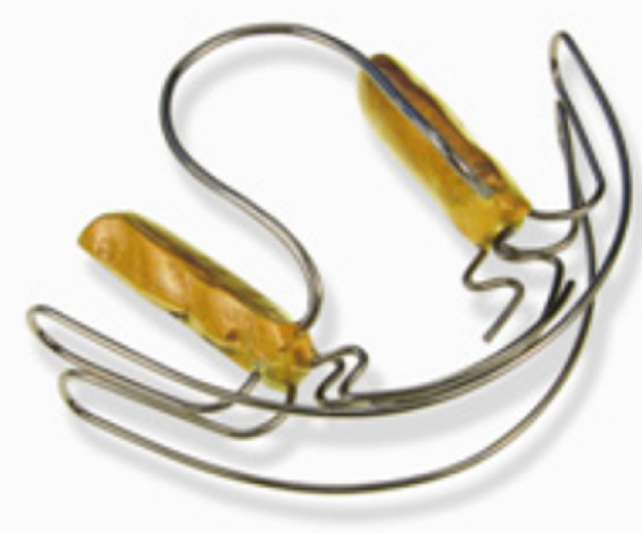

Figura 1. Activador Abierto Elástico de Klammt (AAEK) Fuente: [20]

la lengua puede realizar sus movimientos funcionales y facilitar el habla; por lo tanto, se puede usar de día y de noche [20].

Los cambios de postura y modificaciones programadas de avance mandibular por etapas, realizados a través de los diversos tratamientos $[23,24]$, requieren de una mordida constructiva que determinará la cantidad de desplazamiento mandibular sagital y vertical del activador abierto $[25,26]$.

El objetivo del estudio es identificar los cambios en el complejo craneofacial por medio de radiografías laterales de cráneo de once pacientes con maloclusión clase II división 1, los cuales fueron sometidos a tratamiento de ortopedia funcional con AAEK.

\section{Materiales y métodos}

La finalidad de este estudio fue evaluar cambios cefalométricos en el complejo craneofacial y en tejidos blandos de pacientes con maloclusión clase II división 1 tratados con un AAEK.

De un grupo de veinte pacientes que presentaban maloclusión clase II-1, se seleccionó una muestra de once pacientes, cinco hombres y seis mujeres en edades entre 8 y 12 años.

Se incluyeron todos los pacientes sanos, con dentición mixta, sin caries dental, sin ausencias congénitas, ni anomalías dentales que requerían tratamiento de ortopedia funcional, y se excluyeron todos los pacientes que no cumplieron con estos criterios.
A todos los pacientes seleccionados se les tomaron ayudas diagnósticas que incluían radiografías panorámica y lateral de cráneo, modelos de estudio y estudio fotográfico completo. Todos estos exámenes se tomaron en la Unidad de Radiología Oral y Maxilofacial de la Escuela de Odontología de la Universidad del Valle, Cali, Valle del Cauca, Colombia.

La muestra seleccionada fue tratada con aparatología ortopédica funcional utilizando el AAEK, la cual fue elaborada en el mismo laboratorio en acrílico de autocurado, que unía los elementos metálicos fabricados con alambre de acero inoxidable calibre $0,9 \mathrm{~mm}$ o 0,036 . Se confeccionaron los arcos vestibulares superior e inferior, resortes palatinos y linguales para controlar y guiar la erupción de los incisivos, y resorte tipo coffin que unía los dos componentes laterales. La extensión del acrílico fue desde el canino hasta el último molar erupcionado; el poco volumen del aparato y los elementos funcionales básicos permitieron que la lengua realizara sus movimientos funcionales, facilitando así el habla y una mayor cantidad de tiempo en boca.

Se les informó a los padres de los pacientes acerca del tipo de tratamiento, sus ventajas y desventajas, y se les instruyó el correcto uso de la aparatología. El paciente debía usar el activador por entre seis y ocho horas diarias y toda la noche. Además de ello, se les explicaron los cuidados en cuanto a la higiene oral. Los padres de los pacientes firmaron un consentimiento informado para que sus hijos hicieran parte de este estudio. Durante el año de tratamiento, una vez al mes asistían a control, en el que se indagaba a los padres acerca del uso y la frecuencia de uso de la aparatología, y se dejaba registro en la historia clínica.

Se realizaron trazos cefalométricos al comienzo y al año de tratamiento con el mismo operador previamente calibrado. Las medidas angulares y lineales utilizadas para valorar los cambios en el año de tratamiento aparecen reflejadas en la tabla 1.

\section{Análisis estadístico}

Las medidas cefalométricas de los once pacientes, tomadas antes y después del tratamiento, fueron digitalizadas en una hoja de cálculo de Microsoft Excel para realizar el análisis estadístico. Las variables analizadas están dadas en las siguientes escalas de medición: grados, milímetros y/o porcentajes. 
Tabla 1. Definición de medidas cefalométricas trazadas

\begin{tabular}{ll}
\hline SNA & Ángulo formado por el plano Silla Turca-Nasion y Nasion-Punto A. \\
SNB & Ángulo formado por la unión de los planos Silla Turca-Nasion y Nasion-Punto B. \\
s-N/Go-Gn & Ángulo formado por los planos Silla Turca-Nasion y Gonion-Gnation. \\
FH/Go-Gn & Ángulo formado por el plano horizontal de Frankfort y el plano mandibular. \\
AFP & Medida en milímetros que determina la distancia entre Silla Turca-Gonion. \\
AFA & Medida en milímetros que determina la distancia entre Nasion-Mentón. \\
S-Go/N-Me & Relación porcentual que determina el vector de crecimiento mandibular. \\
ANB & Ángulo formado por los planos Nasion-Punto A y Nasion-Punto B. \\
Witts & Medida en milímetros que determina la proyección del punto A en relación con el punto B sobre el plano oclusal. \\
$\mathbf{1 / B a p}$ & Ángulo formado por el plano biespinal y el eje longitudinal del incisivo superior. \\
$\mathbf{1 / G o - G n}$ & Ángulo formado por el plano mandibular y el eje longitudinal del incisivo inferior. \\
$\mathbf{1}$ sup/inf & Ángulo formado por los ejes coronorradiculares de los incisivos superior e inferior. \\
Overjet & Medida en milímetros que evalúa la posición del borde incisal superior a la cara vestibular del incisivo inferior. \\
Sn-Pg/labio sup & Medida en milímetros que evalúa la posición del labio superior en sentido anteroposterior. \\
Sn-Pg/labio inf & Medida en milímetros que evalúa la posición del labio inferior en sentido anteroposterior. \\
\hline
\end{tabular}

Fuente: Elaboración propia

Para comprobar la distribución normal de los datos de cada variable, se utilizó la prueba de normalidad de Kolmogorov-Smirnov antes y después del tratamiento (por separado), con valores $\mathrm{P}<0,05$, con lo cual se demostró que sí hay una distribución normal.

Se realizó una prueba $\mathrm{T}$ Student para datos pareados y para analizar si existían diferencias en las medidas de las variables pre y postratamiento. Una vez agrupados los datos, se analizaron con el programa SPSS ${ }^{\oplus}$ versión 19 para dos muestras relacionadas, ya que los datos están correlacionados por ser una misma variable medida en un mismo sujeto, pero en condiciones diferentes. Se asumió un nivel de significancia de 0,05 para el análisis estadístico.

\section{Resultados}

La muestra estaba conformada por once pacientes, cinco hombres y seis mujeres, de 8-12 años de edad, con un promedio de edad de 10 años y tres meses, los cuales fueron sometidos a tratamiento de ortopedia maxilar para corregir la maloclusión clase II-1 con un AAEK en un periodo de doce meses.
La tabla 2 presenta los valores de la media, la desviación estándar (DE) y P de los grupos pretratamiento y postratamiento para cada una de las medidas cefalométricas empleadas en este estudio. Las medidas ANB, 1/Bap, 1 sup/1 inf y overjet presentaron diferencias estadísticamente significativas de $\mathbf{P}<\mathbf{0 , 0 5}$.

En general, las medidas de sNA, s-N/Go-Gn, FH/Go-Gn, 1/Go-Gn y la relación porcentual que determina el vector de crecimiento mandibular (S-Go/N-Me) permanecieron casi estables durante el periodo de tratamiento. Otras medidas cefalométricas que presentaron cambios mínimos con incidencia favorable en la corrección de la maloclusión clase II-1 después de realizado el tratamiento con un AAEK fueron: SNB, Witts, y Sn-Pg/Labio Sup y Sn-Pg/Labio Inf (estas dos últimas corresponden a la medida en milímetros que evalúa la posición del labio superior e inferior en sentido anteroposterior, respectivamente).

La figura 2 presenta una radiografía lateral de cráneo inicial y otra postratamiento del mismo paciente, en la que se puede observar una disminución en la protrusión y proinclinación dental después de un año de tratamiento. 
Tabla 2. Media y DE de los valores cefalométricos al inicio y un año después de tratamiento con AAEK

\begin{tabular}{|c|c|c|c|c|c|}
\hline \multirow{2}{*}{ Análisis cefalométrico } & \multicolumn{2}{|c|}{ Grupo preratamiento } & \multicolumn{2}{|c|}{ Grupo postratamiento } & \multirow{2}{*}{$\mathbf{P}$} \\
\hline & Media & ${ }^{*} \mathbf{D E}$ & Media & ${ }^{*} \mathrm{DE}$ & \\
\hline SNA $\left({ }^{\circ}\right)$ & 82,00 & 3,975 & 81,64 & 4,105 & 0,476 \\
\hline SNB $\left({ }^{\circ}\right)$ & 75,73 & 3,101 & 76,73 & 3,495 & 0,067 \\
\hline s-N/Go-Gn $\left(^{\circ}\right)$ & 34,36 & 3,355 & 34,18 & 4,119 & 0,800 \\
\hline FH/Go-Gn $\left({ }^{\circ}\right)$ & 23,73 & 4,519 & 24,27 & 3,690 & 0,684 \\
\hline $\operatorname{AFP}(\mathbf{m m})$ & 67,73 & 3,717 & 70,00 & 5,568 & 0,240 \\
\hline AFA $(\mathbf{m m})$ & 108,73 & 6,436 & 111,64 & 8,698 & 0,375 \\
\hline S-Go/N-Me (\%) & 0,623 & 0,0262 & 0,627 & 0,0310 & 0,637 \\
\hline ANB $\left({ }^{\circ}\right)$ & 6,27 & 1,489 & 4,73 & 2,102 & $0,003^{* *}$ \\
\hline Witts (mm) & 2,00 & 1,8974 & 0,64 & 2,618 & 0,091 \\
\hline 1/Bap $\left(^{\circ}\right)$ & 120,09 & 4,549 & 107,91 & 6,252 & $0,000^{\star *}$ \\
\hline 1/Go-Gn $\left({ }^{\circ}\right)$ & 96,82 & 8,542 & 96,45 & 4,947 & 0,832 \\
\hline $1 \sup / \inf \left(^{\circ}\right)$ & 116,82 & 9,042 & 129,09 & 7,582 & $0,000^{\star *}$ \\
\hline Overjet (mm) & 8,730 & 2,149 & 3,591 & 0,970 & $0,000^{\star *}$ \\
\hline Sn-Pg/labio sup (mm) & 5,55 & 2,583 & 4,23 & 1,403 & 0,035 \\
\hline Sn-Pg/labio inf (mm) & 4,36 & 2,501 & 3,68 & 1,765 & 0,176 \\
\hline
\end{tabular}

${ }^{*}$ DE: Desviación estándar. ${ }^{* *}$ Diferencia significativa $(\mathrm{p}<0,05)$.

Fuente: elaboración propia
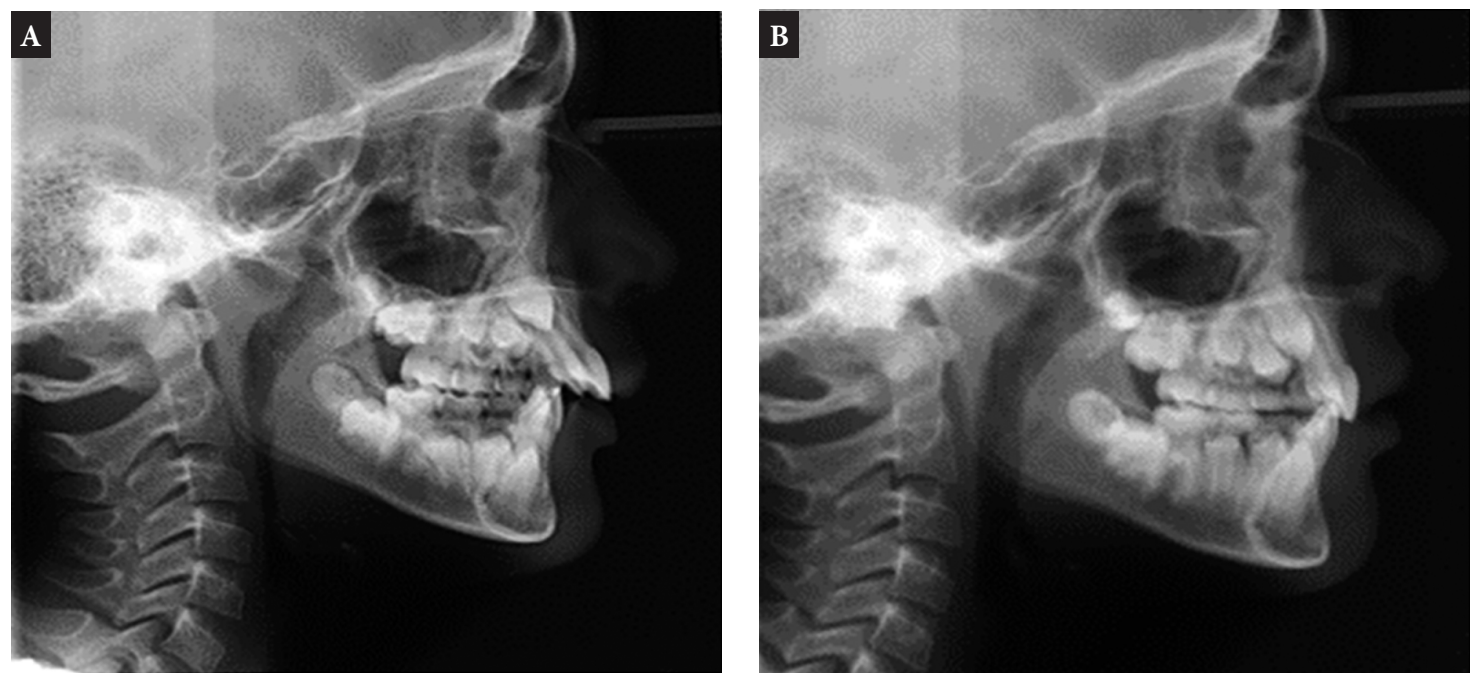

Figura 2. A. Radiografía lateral de cráneo inicial. B. Radiografía lateral de cráneo un año después de tratamiento con AAEK Fuente: elaboración propia

\section{Discusión}

En la literatura, se cuestiona si la terapia ortopédica funcional produce una modificación definitiva en la tendencia de crecimiento o si solo la redirecciona, y si una vez retirada la aparatología, el crecimiento de los maxilares vuelve a quedar supeditado a un fuerte control genético que cambie desfavorablemente el biotipo facial de los pacientes tratados. Los aparatos ortopédicos funcionales tienen como mecanismo característico sostener la mandíbula en posición adelantada, durante las horas de uso, y se ha proclamado su capacidad de estimular el crecimiento mandibular [27]. 
Tanto el AAEK como otros diseños tienen el denominador común de forzar el adelantamiento de la mandíbula con la idea de estimular la zona precondroblástica acelerando la formación de condroblastos y condrocitos que dan como resultado el crecimiento condilar; esto acompañado de una remodelación anteroinferior de la fosa glenoidea [28-30] generará una nueva posición mandibular dada por el aparato, la cual se estabiliza y logra la corrección de la maloclusión clase II [27].

Los efectos que producen los aparatos ortopédicos funcionales varían también por razones relacionadas con la genética, factores ambientales o extrínsecos, el conocimiento del profesional, la respuesta al tratamiento de cada paciente y la colaboración tanto de los niños como de los padres. Diferentes estudios han demostrado que el AAEK es una alternativa terapéutica eficaz para la corrección de las distoclusiones, permitiendo el avance mandibular y favoreciendo cambios dentoalveolares en niños clase II-1 $[20,31]$.

En este estudio, se observó de forma significativa una mejoría en la relación maxilomandibular, principalmente a través de los cambios obtenidos en SNB, ANB, Witts y overjet después de un año de tratamiento ortopédico con un AAEK. La muestra de este estudio estaba conformada por un grupo de pacientes en crecimiento y en periodo de dentición mixta; el proceso del desarrollo influye tanto en la etiopatogenia como en la corrección de la maloclusión, por lo cual todo lo que contribuya para lograr un avance $o$ adelantamiento mandibular será favorable. El principal objetivo terapéutico se centra entonces en promover el avance de la arcada inferior para lograr relaciones oclusales óptimas a nivel molar, a nivel de caninos y a nivel de resalte y sobremordida vertical [32, 33].

En el caso de la influencia sobre el maxilar (sNA), este estudio coincide con el de Pérez García [34], Courtney [35] y otros autores, quienes refieren una tendencia a la restricción de su crecimiento durante el tratamiento con aparatología funcional. En este estudio, se pueden observar cambios a nivel del ángulo SNB de 1 y $2^{\circ}$ que inciden positivamente en la corrección de la maloclusión, lo que resulta en una mejora del ángulo ANB en pacientes con maloclusión clase II-1.

Con aparatología ortopédica tipo Headgear, se ha encontrado una pequeña restricción en el crecimiento hacia adelante del maxilar superior con disminución del ángulo sNA de entre 0,5 y $3^{\circ}$ [12].
En este estudio, se pudo observar restricción en el crecimiento hacia adelante del maxilar (SNA) y un posicionamiento anterior del punto $\mathrm{B}$ (aumento de SNB entre 1 y $2^{\circ}$ ), lo que resulta en una mejora del ángulo ANB aproximadamente de 1 y $3^{\circ}$ después de un promedio de doce meses de tratamiento con Un AAEK.

Jakobsson [36], en su estudio, realizó el seguimiento de tres grupos de niños clase II, diecinueve pacientes por grupo, con un promedio de edad de 8,5 años; estos fueron asignados aleatoriamente a dieciocho meses de tratamiento con Headgear de tracción cervical y a tratamiento con activador de Andresen. El grupo de Headgear restringió el punto A aproximadamente $1,6 \mathrm{~mm}$ y distalizó molares superiores un promedio de $3,7 \mathrm{~mm}$ en comparación con el grupo control. El grupo del activador restringió el punto A un promedio de $0,7 \mathrm{~mm}$ y distalizó los molares superiores un promedio de 1,2 mm en comparación con el grupo control. Una reducción significativa en el overjet fue encontrada con ambos grupos, teniendo el grupo del activador la mayor reducción. No hubo cambios significativos en la longitud mandibular en ninguno de los grupos [12]. Los resultados de este estudio realizado con otro tipo de aparatología ortopédica funcional concuerdan con los resultados aquí obtenidos, en los que se puede observar que el AAEK produce cambios significativos en: ANB, 1/Bap, 1 sup/1 inf y overjet.

La convexidad facial disminuyó en este estudio, lo que muestra cambios radiográficamente significativos. La reducción de la convexidad facial puede atribuirse a la estabilidad del punto A y al avance del punto Pogonion después del tratamiento $[20,37]$. Un perfil facial armónico es el reflejo de las proporciones ideales entre las distintas áreas de la cara. Ellas dependen de las proporciones dentarias, óseas y de los tejidos blandos [38].

\section{Conclusiones}

En este estudio, se observó que el tratamiento temprano de la maloclusión clase II-1 en niños en crecimiento con un AAEK produjo cambios cefalométricos favorables a nivel esquelético, dental y de tejidos blandos, que permitieron alcanzar los objetivos propuestos.

Estos cambios indican: inhibición del crecimiento anterior del área dentoalveolar superior, 
verticalización de los incisivos superiores, restricción de la migración anterior de los molares superiores y posicionamiento anterior de la mandíbula reflejado con un valor mayor del ángulo sNB. El patrón de crecimiento no sufrió modificaciones con el tratamiento.

Factores genéticos, ambientales, de tipo de dentición, de patrón de crecimiento, de edad y de periodo de crecimiento, entre otros, tienen un papel fundamental en las variaciones de los resultados obtenidos después de un tratamiento de ortopedia maxilar.

Entre la amplia gama de aparatología ortopédica funcional para corrección de maloclusiones clase II, el AAEK se presenta como una excelente opción para el tratamiento temprano de este tipo de maloclusión, ya que permite obtener muy buenos resultados en un periodo corto de tiempo, mejorar la función oclusal y la estética, y disminuir problemas periodontales en pacientes con maloclusión clase II-1.

\section{Referencias}

[1] Rodríguez C, Padilla MR. Manejo temprano de la maloclusión clase II división 2. Revisión de la literatura. Rev Estomatol. 2015;23(2):57-63.

[2] Rothstein T, Yoon-Tarlie C. Dental and facial skeletal characteristics and growth of males and females with Class II, Division 1 malocclusion between the ages of 10 and 14 (revisited) - Part I: Characteristics of size, form, and position. Am J Orthod Dentofacial Orthop. 2001;120(5):542-55.doi: http:// dx.doi.org/10.1067/mod.2001.118628

[3] Canut JA. Ortodoncia clínica. Barcelona: Salvat; 1989.

[4] White W. Introducción a la ortodoncia. Barcelona: Interamericana; 1991.

[5] Ustrell T, Joseph M. Ortodoncia. 2. edición. Barcelona: Ediciones Universitat; 2002.

[6] Lugo C, Toyo I. Hábitos orales no fisiológicos más comunes y cómo influyen en las maloclusiones. Revista Latinoamericana de Ortodoncia y Odontopediatría [internet]. 2011 [consultado 2015 dic 10]. Disponible en: https://www.ortodoncia.ws/publicaciones/2011/art-5/

[7] Echarri P, Carrasco A, Vila Manchó E, Bottini E. Protocolo de exploración interdisciplinar orofacial para niños y adolescentes. Ortod Esp. 2009;49(2):107-15.

[8] Bottini E, Carrasco A, Echarri P, Molina A, Padrós E, Mascó JP, et al. Protocolo de exploración interdisciplinaria orofacial para adultos: manejo y codificación. Rev Esp Ortod. 2011;41(2):71-8.
[9] McLain JB, Proffit WR. Oral health status in the United States: Prevalence of malocclusion. J Dent Educ. 1985;49(6):386-97.

[10] Álvarez CA. Frecuencia de los componentes de la maloclusión clase iI esquelética en dentición mixta. Medigraphic. 2006;63(6):210-4.

[11] Bishara SE. Class II malocclusions: Diagnostic and clinical considerations with and without treatment. Semin Orthod. 2006;12(1):11-24. doi: http://doi. org/10.1053/j.sodo.2005.10.005

[12] Southard TE, Marshall SD, Allareddy V, Moreno LM, Holton NE. An evidence-based comparison of Headgear and functional appliance therapy. Semin Orthod. 2013;19(3):174-95. doi: http://dx.doi. org/10.1053/j.sodo.2013.03.007

[13] Ramírez G. Razonamiento biológico para el tratamiento temprano. En: Tratamiento temprano de las maloclusiones: prevención e intercepción en dentición primaria. Cúcuta: Talleres Gráficos; 2009. p. 27-34.

[14] Hernández J, Padilla M. Tratamiento temprano de la mordida cruzada anterior. Revisión de literatura. Rev Estomat. 2011;19(2):40-7.

[15] McNamara JA, Brudon WL. Orthodontics and dentofacial orthopedics. Ann Arbor: Needham Press; 2001.

[16] Petrovic A, Stutzmann J, Lavergne J. Mechanism of craniofacial growth and modus operandi of functional appliances: A cell-level and cybernetic approach to orthodontic decision making. In: Craniofacial growth theory and orthodontic treatment. Ann Arbor: Center for Human Growth and Development, University of Michigan; 1990.

[17] Woodside DG, Metaxas A, Altuna G. The influence of functional appliance therapy on glenoid fossa remodeling. Am J Orthod Dentofacial Orthop. 1987;92(3):181-98. doi: https://doi.org/10.1016/088 9-5406(87)90411-2

[18] Shum L, Rabie ABM, Hagg U. Vascular endothelial growth factor expression and bone formation in posterior glenoid fossa during stepwise mandibular advancement. Am J Orthod Dentofacial Orthop. 2004;125(2):185-90. doi: http://dx.doi.org/ 10.1016/j.ajodo.2002.12.002

[19] Bedoya A, Franco G. Tratamiento de maloclusión clase II con aparatología ortopédica funcional: Bionator. Reporte de un caso. Rev Estomat. 2010;18 (1):24-9.

[20] Bedoya A, Chacón A. Tratamiento temprano de maloclusiones clase II tratado con Activador Abierto Elástico de Klammt (AAEK). Reporte de caso. Rev Estomat. 2009;17(2):23-9. 
[21] Saldarriaga JA, Álvarez E, Botero PM. Tratamientos para la maloclusión clase II esquelética combinada. Rev CEs Odont. 2013;26(2):145-59.

[22] Gonçalve RC, Meloti AF, Gandini Jr. LG, Raveli DV, Pinto ADS. Alterações dentoesqueléticas verticais e horizontais induzidas pelo ativador elástico Aberto de Klammt. Rev Ortodontia. 2011;44(5):460-6.

[23] Pérez LM, Saenz ML, Castillo R, Soto L, Grau R. Adaptación funcional con el uso del activador de Klammt relacionada con el grado de protrusión mandibular. Rev Cubana Ortod. 2001;16(2):96-101.

[24] Rabie ABM. Does the degree of advancement during functional appliance therapy matter? Eur J Orthod. 2008;30(3):274-82. doi: https://doi.org/10.1093/ejo/ cjm 129

[25] BisharaSE,Ziaja RR. Functionalappliances: A review. AmJOrthodDentofacialOrthop. 1989;95(3):250-58. doi: https://doi.org/10.1016/0889-5406(89)90055-3

[26] Skay J. Construction bite. How important is it? Inter J Orthod. 1984;22(2-3):19.

[27] Proffit WR, Fields HW. Planificación del tratamiento ortodóncico: limitaciones, controversias y problemas especiales. En: Ortodoncia contemporánea, teoría y práctica. 3. ${ }^{a}$ edición. Madrid: Harcourt; 2002.

[28] Graber TR, Petrovic T. A orthodontic and dentofacial orthopedic treatment. $5^{\text {th }}$ edition. St Louis: Mosby; 2009 .

[29] Araujo AM, Buschang PH, Melo AC. Adaptive condylar growth and mandibular remodelling changes with bionator therapy. An implant study. Eur J Orthod. 2004;26(5):515-22. doi: https://doi.org/10.1093/ ejo/26.5.515

[30] Rabie AB, Zhao Z, Shen G, Hagg EU, Robinson W. Osteogenesis in the glenoid fossa in response to mandibular advancement. Am J Orthod Dentofacial Orthop. 2001;119(4):390-400. doi: http://doi. org/10.1067/mod.2001.112875
[31] Curbeira Hernández EM, Mora Pérez CC. Activador abierto elástico de Klammt y bloques gemelos en el tratamiento del síndrome de clase II. Rev MediSur. 2009;7(1):114-23.

[32] Pérez López M, Fernández Ysla R, Otaño Laffitte G, Delgado Carrera L. Cambios cefalométricos esqueletales y de tejidos blandos en pacientes tratados con bloques gemelos. Revista Habanera de Ciencias Médicas. 2005;4(3):1-15.

[33] Rothstein T, Phan XL. Dental and facial skeletal characteristics and growth of females and males with Class II Division 1 malocclusion between the ages of 10 and 14 (revisted). Part II: Anteroposterior and vertical circumpubertal growth. Am J Orthod Dentofacial Orthop. 2001;120(5):542-55. doi: http:// doi.org/10.1067/mod.2001.118628

[34] Pérez García L, Saez Luna M, Castillo Hernández R, Soto Cantero L, Grau Ávalo R. Cambios cefalométricos con el uso del activador de Klammt en diferentes magnitudes de protrusión mandibular. Rev Cubana Estomatol. 2003;40(2):32-5.

[35] Courtney M, Harkness M, Herbison P. Maxillary and cranial base changes during treatment with functional appliances. Am J Orthod Dentofacial Orthop. 1996;109(6):216-24. doi: http://doi. org/10.1016/S0889-5406(96)70073-2

[36] Jakobsson SO. Cephalometric evaluation of treatment effect on Class II, Division 1 malocclusions. Am J Orthod. 1967;53(6):446-57. doi: https://doi. org/10.1016/0002-9416(67)90005-X

[37] Fernández R, Marín G, Otaño G, Pérez M, Delgado L. Los bloques gemelos. Uso y construcción del aparato convencional. Rev Cubana Estomatol. 2005;42(3) [internet]. Disponible en: http://scielo. sld.cu/scielo.php?script=sci_arttext\&pid=S003475072005000300007

[38] Gregoret J. Análisis lateral de Ricketts. En: Ortodoncia y cirugía ortognática. Diagnóstico y planificación de tratamiento. Barcelona: Publicaciones Médicas; 1997. 